\title{
Transient gelation by spinodal decomposition in colloid-polymer mixtures
}

\author{
Nynke A.M. Verhaegh ${ }^{\mathrm{a}, *}$, Daniela Asnaghi ${ }^{\mathrm{b}}$, \\ Henk N.W. Lekkerkerker ${ }^{\mathrm{a}}$, Marzio Giglio ${ }^{\mathrm{b}}$, Luca Cipelletti ${ }^{\mathrm{b}}$ \\ a Van't Hoff Laboratory, Utrecht University, Padualaan 8, $3584 \mathrm{CH}$ Utrecht. The Netherlands \\ b Department of Physics and INFM, University of Milan, Via Celoria 16, 20133 Milan, Italy
}

Received 26 June 1996; revised 31 January 1997

\begin{abstract}
We have investigated with small angle light scattering and optical microscopy transient gelation phenomena which occur in phase-separating colloid-polymer mixtures. The scattering intensity distribution shows a peak at non-zero wave vector and satisfies the asymptotic $q^{-4}$ Porod behaviour. Consistent with these observations, optical micrographs show an alternating pattern of dark and bright domains. These findings suggest that the polymer-induced depletion forces lead to the formation of a bicontinuous network of colloid-rich and colloid-poor domains, via a spinodal decomposition process. This bicontinuous network rapidly attains a gellike character as indicated by the arrest of speckle fluctuations. The occurrence of the gel is ascribed to polymer-induced aggregation between the colloids in the colloid-rich phase. Due to the reversible nature of the aggregation the network restructures and eventually the gel collapses, as is manifested by the rapid separation of the colloid-rich phase from the colloid-poor phase.
\end{abstract}

\section{Introduction}

It is well-established that the depletion attraction between colloidal particles induced by the addition of non-adsorbing polymers, may give rise to phase separation in a colloidal suspension. The depletion-induced attraction was first described by Asakura and Oosawa [1] and Vrij [2]. Colloids are surrounded by a depletion zone from which the polymers are sterically excluded. When the colloids are close enough there is an overlap of depletion zones, causing an unbalanced osmotic force. This results in an attractive interaction between the colloidal particles, the range and depth of which can be tuned by the volume fraction $\left(\phi_{\mathrm{p}}\right)$ and the diameter $\left(\sigma_{\mathrm{p}}\right)$ of the polymer, giving rise to different types of phase diagrams $[3,4]$. For short-range attractions $\left(\sigma_{\mathrm{p}} / \sigma_{\mathrm{c}}<0.3\right.$,

\footnotetext{
${ }^{*}$ Corresponding author.
} 
where $\sigma_{\mathrm{c}}$ is the diameter of the colloid) the region of fluid-crystal coexistence is expanded. For long ranges of attraction $\left(\sigma_{\mathrm{p}} / \sigma_{\mathrm{c}}>0.3\right)$ a rich phase-behaviour is encountered including two-phase regions (gas-liquid, gas-solid and liquid-solid) and a three-phase region (gas-liquid-solid). These theoretical predictions for the phase diagram have been experimentally confirmed [5-7].

Recent studies have focussed on the phase separation kinetics in colloid-polymer mixtures both with a relatively long-range attraction [8] and with a relatively short range attraction [9-11]. In the former case $\left(\sigma_{\mathrm{p}} / \sigma_{\mathrm{c}} \approx 1\right)$ fluid-fluid phase separation was observed. The kinetics could be described with theories developed for phase separation in binary liquid mixtures. For the short-range attraction $\left(\sigma_{\mathrm{p}} / \sigma_{\mathrm{c}} \approx 0.08\right)$ different kinds of kinetic behaviour were mapped into the phase diagram by Poon, Pusey and coworkers [10]. If there is enough polymer present, the system undergoes a fluid-crystal phase separation. For larger amounts of polymer a metastable fluid-fluid phase boundary is crossed giving rise to phase separation following nucleation and growth or spinodal decomposition. Further addition of polymer leads to transient gelation. Initially the colloids form a space-spanning network with frozen dynamics, but internal restructuring allows for a final collapse of the gel. A scattering peak was observed at non-zero wave vector in the small angle light scattering profile from these transient gels [10,11]. The time resolved intensity distributions satisfied dynamic scaling with an exponent 1.7 , the fractal dimension of aggregates formed under Diffusion Limited Cluster Aggregation (DLCA) conditions (aggregation with infinitely deep potential) [12].

In this paper we study colloid-polymer mixtures with a size ratio colloid over polymer $\sigma_{\mathrm{p}} / \sigma_{\mathrm{c}}$ equal to $\frac{1}{4}$ and a polymer volume fraction in the range of $40 \% \leqslant \phi_{\mathrm{p}} \leqslant 85 \%$ by means of small angle light scattering and optical microscopy. Using a simple expression for the well depth $(W)$ of the depletion attraction $[1,2]$,

$$
\frac{W}{k_{\mathrm{B}} T}=-\phi_{\mathrm{p}} \frac{3}{2} \frac{\sigma_{\mathrm{c}}}{\sigma_{\mathrm{p}}},
$$

it can be seen that the system under study has a well depth in the order of 2.5 $5 k_{\mathrm{B}} T$ ( $k_{\mathrm{B}}$ is the Boltzmann constant and $T$ is the temperature), allowing for the occurrence of aggregation and phase separation. The system studied here displays transient gelation even although its depletion attraction is much shallower than for the system studied by Pusey, Poon and coworkers [9-11]. We find experimental indications that gelation in the present system is a consequence of spinodal decomposition. The possibility of network formation and hence gelation in colloidal dispersions by spinodal decomposition was already discussed more than ten years ago [13]. This mechanism of gelation was demonstrated convincingly in the case of demixing polymer solutions $[14,15]$ but as far as we know no experimental evidence for spinodal decomposition induced gelation has been reported for the case of unstable colloidal dispersions.

The paper is organized as follows. In Section 2 we introduce the system and the experimental techniques. In Section 3 we present the experimental results which are discussed in Section 4. We draw conclusions in Section 5. 


\section{Materials and methods}

\subsection{Sample description}

We used silica spheres which were nucleated in microemulsions as is described by Osseo-Asare and Arriagada [16,17]. The nuclei were grown to a diameter of $184 \mathrm{~nm}$ (as determined with static and dynamic light scattering) following the procedures by Van Blaaderen [18], and finally they were sterically stabilized by coating with stearyl alcohol [19]. The polydispersity (standard deviation divided by mean diameter) equals $5 \%$ and the density $1.68 \mathrm{~g} \mathrm{ml}^{-1}$. As polymers we used polydimethylsiloxane (PDMS, Janssen) with a mean radius of gyration of $23 \mathrm{~nm}$ and an average molecular weight $M_{\mathrm{w}}$ of $2.11 \times 10^{5} \mathrm{~g} / \mathrm{mol}$ as determined by gel permeation chromatography and static light scattering (Zimm plots). The solvent was cyclohexane (Janssen) in which both the colloids and the polymers are practically refractive index matched. Transient gels were studied in mixtures with colloid volume fractions $\left(\phi_{\mathrm{c}}\right)$ around $10 \%$ and concentration polymers $\left(c_{\mathrm{p}}\right)$ between 2.75 and $5.75 \mathrm{mg} / \mathrm{ml}$. Defining the polymer volume fraction $\phi_{\mathrm{p}}$ as the polymer number density times a spherical volume with radius equal to the radius of gyration of the polymer, this corresponds to $40 \% \leqslant \phi_{\mathrm{p}} \leqslant 85 \%$. The experiments were performed at room temperature which is well above the $\theta$-temperature of PDMS in cyclohexane $\left(-80^{\circ} \mathrm{C}\right)$. Prior to measurements the mixtures were shaken vigorously in order to be homogenized.

Fig. 1 shows a partial phase diagram, which was established by studying a large number of mixtures. Mixtures in the stable region of the phase diagram slowly settled (in weeks) into a dense sediment that showed purple Bragg reflections in the backward scattering direction. It appeared to be difficult to locate the phase boundary

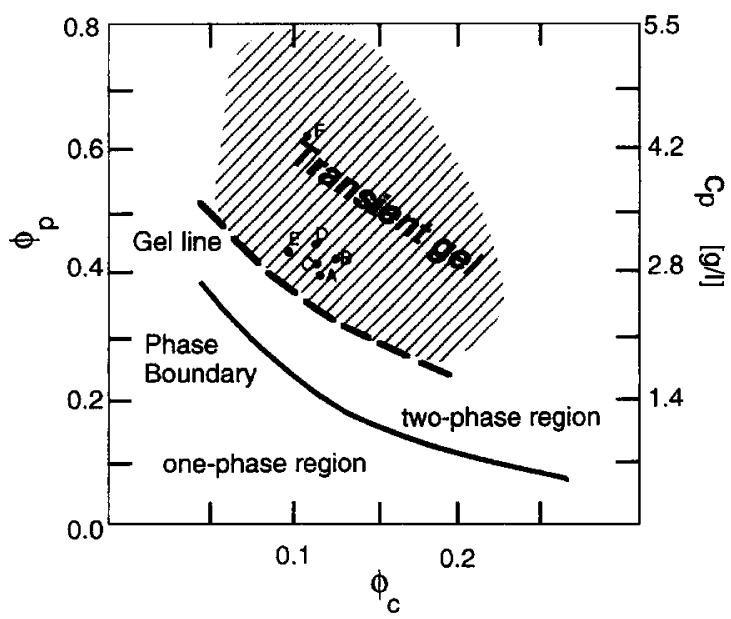

Fig. 1. Phase diagram for the colloid-polymer mixtures under study. The phase boundary and the gel line as determined by visual inspection, are represented. The studied samples are indicated by the points labelled A, B, C, D, E, F the compositions of which are given in Table 1 . 
Table 1

Parameters of the mixtures under study. $\phi_{\mathrm{c}}$ and $\phi_{\mathrm{p}}$ are, respectively, the colloid and polymer volume fraction, $c_{\mathbf{p}}$ is the polymer concentration, $\alpha$ is the decay exponent of the scattering distribution at large wave vector, $t_{\mathrm{gel}}$ is the life time of the gel and $q_{\mathrm{m}}^{\mathrm{gel}}$ is the wave vector corresponding to the scattering peak when the gel is formed

\begin{tabular}{lllllrl}
\hline & $\begin{array}{l}\phi_{\mathrm{c}} \\
(\%)\end{array}$ & $\begin{array}{l}\phi_{\mathrm{p}} \\
(\%)\end{array}$ & $\begin{array}{l}c_{\mathrm{p}} \\
(\mathrm{mg} / \mathrm{ml})\end{array}$ & $\alpha$ & $\begin{array}{l}t_{\mathrm{gel}} \\
(\mathrm{s})\end{array}$ & $\begin{array}{l}q_{\mathrm{m}}^{\mathrm{gel}} \\
\left(\mathrm{cm}^{-1}\right)\end{array}$ \\
\hline $\mathrm{A}$ & 0.1151 & 0.3989 & 2.75 & $4.0+0.05$ & 22 & - \\
$\mathrm{B}$ & 0.1226 & 0.4410 & 3.04 & $4.1+0.1$ & 40 & - \\
$\mathrm{C}$ & 0.1135 & 0.4293 & 2.96 & $4.0+0.1$ & 66 & 2650 \\
$\mathrm{D}$ & 0.1135 & 0.4797 & 3.31 & $3.8+0.05$ & 120 & 3150 \\
$\mathrm{E}$ & 0.0966 & 0.4595 & 3.17 & $3.9+0.05$ & 52 & 3300 \\
$\mathrm{~F}$ & 0.1077 & 0.6356 & 4.39 & $3.1+0.1$ & 202 & 3400 \\
$\mathrm{G}$ & 0.0709 & 0.833 & 5.75 & - & 619 & - \\
\hline
\end{tabular}

between the one-phase and two-phase region unambiguously. The boundary determined by dilution of mixtures in the two-phase region, did not correspond precisely with the one determined by concentration of systems in the one-phase region. Mixtures exhibiting two-phase coexistence separated into a dilute upper phase and a dense amorphous bottom phase after about one hour. No crystallites were observed in the bulk of the dense lower phase, but after approximately a week a small top layer showing purple Bragg reflections had grown onto this dense phase. We could not determine the nature of the two phases unequivocally.

The gel transition, which is of primary interest in this work was accurately located by looking at the scattering pattern. Samples exhibiting transient gel behaviour are characterized by a temporary arrest of speckle fluctuations. This gel transition turned out to be highly reproducible. In Table 1 we have listed the compositions of the systems in the gel region which were studied in detail. These samples have also been indicated in Fig. 1.

\subsection{Small angle light scattering set up and light microscopy}

The small angle light scattering set-up employed is similar to the one described in Ref. [20]. A spatially filtered and collimated He-Ne laser beam is focussed by a lens onto a tiny pinhole in the center of a multi-elements sensor array. Before reaching the sensor plane, the beam impinges on the sample and is scattered. The scattered light is detected by 31 annular sensing elements, centered around the pinhole, with radii and thicknesses geometrically increasing. The transmitted beam, passes through the pinhole and is collected by an additional photodiode, placed behind the sensor plane. The signal from this sensor together with the signal from a beam power monitor are used to determine the sample turbidity. This setup can cover up to three decades in transferred momentum $q$, the upper and lower limits of the range in $q$ depending on the 
sample-to-sensor distance. In the present configuration, $q$ spans roughly two decades from $6 \times 10^{2}$ to $5 \times 10^{4} \mathrm{~cm}^{-1}$, the scattering angles varying between $0.2^{\circ}$ and $20^{\circ}$. The phase separating mixtures were also studied using a Zei $\beta$ axioplan microscope in transmission with a $20 \times$ objective. The precise magnification was calibrated by imaging a diffraction grating $(100$ lines $/ \mathrm{mm})$ with the microscope.

Both the time-resolved small angle light scattering and the light microscopy measurements were performed in the same rectangular glass cells of $1 \mathrm{~mm}$ optical path length (Hellma) under similar conditions. Time zero was defined as the moment at which shaking was stopped. The measurements started immediately after homogenization and were continued throughout the collapse of the gel.

\section{Experimental results}

\subsection{General observation}

Fig. 2 gives a pictorial presentation of the dynamics of our samples in the gel region. The observations closely resemble those reported by Pusey et al. for a related system [9]. After homogenization by fierce shaking, we have a slightly turbid mixture for which the scattering pattern consists of speckles fluctuating more rapidly with increasing angles. This angle-dependence of the fluctuation rate is characteristic for diffusing Brownian particles. Furthermore, the scattering pattern displays a bright ring at scattering vector $q_{\mathrm{m}}$ (see Fig. 2(a)). Such a maximum in the scattering profile is typical for systems with a certain degree of order at length scales $A$ roughly corresponding to $2 \pi / q_{\mathrm{m}}$. We have no guarantee that even fierce shaking leads to complete homogenization on the smallest scales in the system. However, we observed that a poor initial homogenization results in strong forward scattering i.e. the absence of a ring. This indicates that the scattering ring is due to the demixing process and not to inhomogeneities that remain in the system in spite of fierce shaking. During approximately the first $10 \mathrm{~s}$ the scattering ring shrinks, indicating that the characteristic length scale of the system is growing. Afterwards, the time-fluctuation of the speckles is almost arrested and the scattering profile exhibits a 'frozen' ring at a fixed wave vector $\left(q_{\mathrm{m}}^{\text {gel }}\right)$ (Fig. $2(\mathrm{~b})$ ). This pattern hardly changes during a period of time, apart from a gradual increase in intensity inside the scattering ring. The speckles display very slow fluctuations which are now hardly angle dependent, as we also verified by video-recording the fluctuation of the speckle intensity simultaneously at two different angles. After a distinct period, which we shall refer to as the gel life time $\left(t_{\text {gel }}\right)$, suddenly the scattering ring degenerates into strong forward scattering (Fig. 2(c)), and speckles start fluctuating rapidly in a typical diffusive mode. At the same time we observe an interface which goes down with a rate of about $10 \mathrm{~mm} / \mathrm{h}$ for all studied samples. For comparison, without added polymer it takes the colloidal particles about a week to sediment over a distance of $10 \mathrm{~mm}$. The colloid-rich phase settles into a dense sediment with volume fractions $\phi_{\mathrm{c}}$ of the order of $50 \%$. These sediments do not show any sign of crystallinity. 


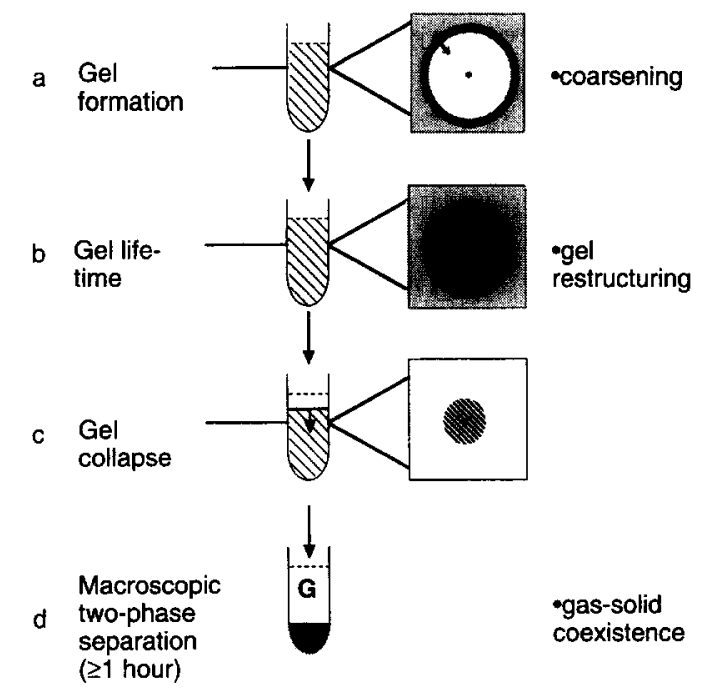

Fig. 2. Schematic representation of the unmixing phenomenon as observed in a cuvette and the corresponding light scattering patterns as projected on a screen, at different instants during the process. (a) gel formation, (b) gel lifetime, (c) gel collapse, (d) macroscopic 2-phase separation.

We found that samples prepared with a larger amount of polymer have longer gel life times (see also Table 1). Similarly an increase in colloid volume fraction also slows down the collapse of the gel. However, this is not the subject of the present paper.

\subsection{Small angle light scattering}

Fig. 3 shows a set of time-resolved scattering curves (a) together with the timeevolution of the sample turbidity (b), for mixture $E\left(\phi_{\mathrm{c}}=9.7 \%\right.$ and $\left.\phi_{\mathrm{p}}=46 \%\right)$. The features shown in this figure are common to all the samples studied and listed in Table 1. In the initial stage, when the gel is formed, a scattering peak at non-zero wave vector can be seen, which shifts to smaller wave vector and increases slightly in intensity. Simultaneously, the sample turbidity also slightly increases. After this initial stage, which lasts only for a few seconds, the shift of the peak is arrested. A similar evolution of the intensity peak position was observed by Verduin and Dhont [21] in an adhesive-hard sphere dispersion. The gelation time in our system is so short, probably due to the small size of the particles involved, that we cannot check whether dynamic scaling holds.

During the gel life time the scattering peak stays at a fixed position which is listed in Table 1 as $q_{\mathrm{m}}^{\text {gel }}$. Note that $q_{\mathrm{m}}^{\text {gel }}$ is in the order of $3000 \mathrm{~cm}^{-1}$, roughly corresponding to a characteristic length scale $\Lambda^{\text {gel }}$ of $20 \mu \mathrm{m}$. The turbidity of the samples stays at a plateau value. However, the intensity at the small wave vector side of the peak increases slightly. At large wave vector all light scattering curves collapse onto the same asymptotic curve which displays a $q^{-\alpha}$ behaviour. For most the samples studied 

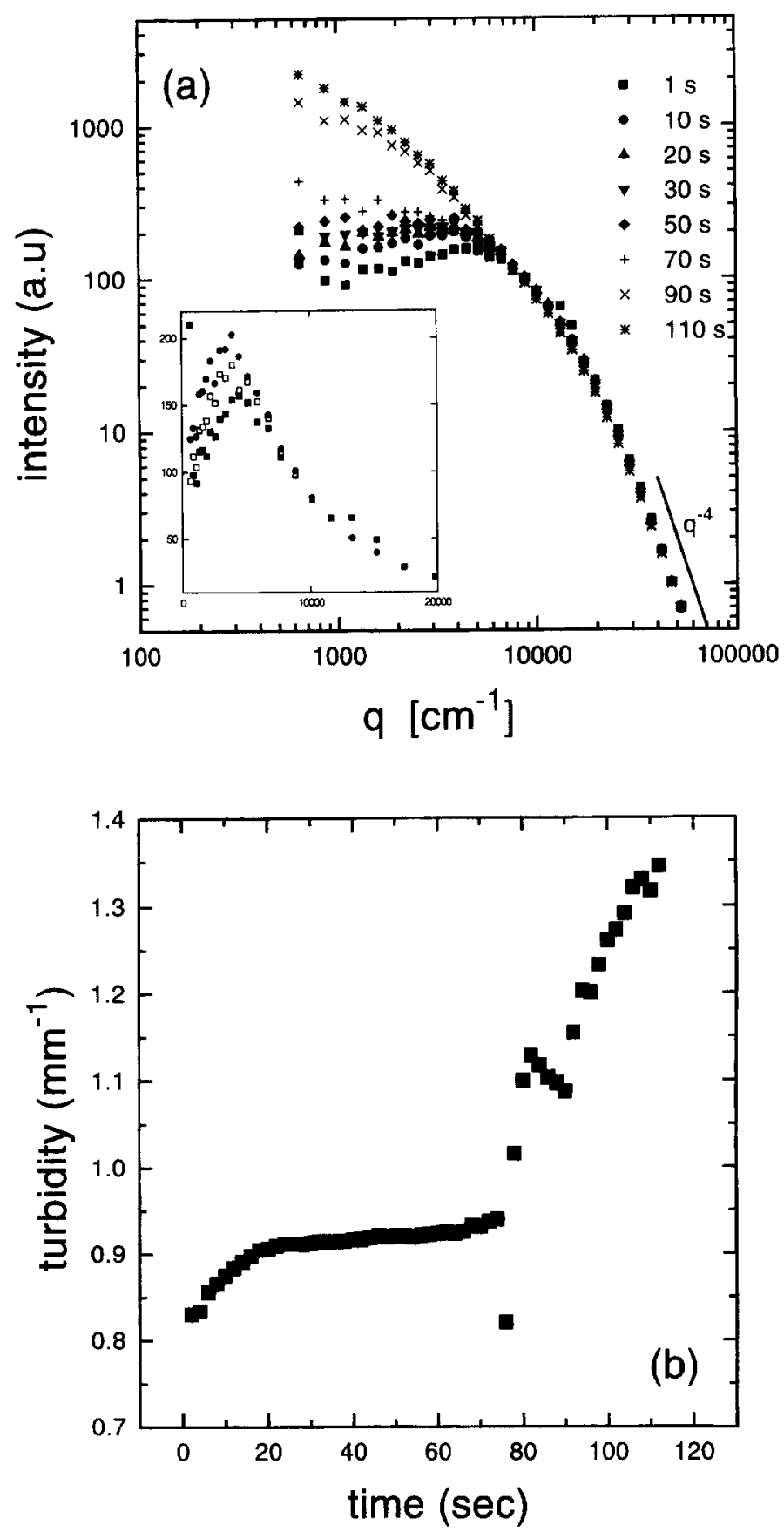

Fig. 3. (a) Time evolution of small angle light scattering profile for a representative mixture with $\phi_{\mathrm{p}}=0.46$ and $\phi_{\mathrm{c}}=0.097$. The time $t=0$ corresponds to the homogenization of the system. The curve $q^{-4}$ is also shown for comparison with the scattering data in the high $q$ region. Three early scattering curves $(t=1$, 5 and $10 \mathrm{~s}$ ) are shown on a lin-lin plot in the inset. (b) The corresponding time evolution of the sample turbidity. 


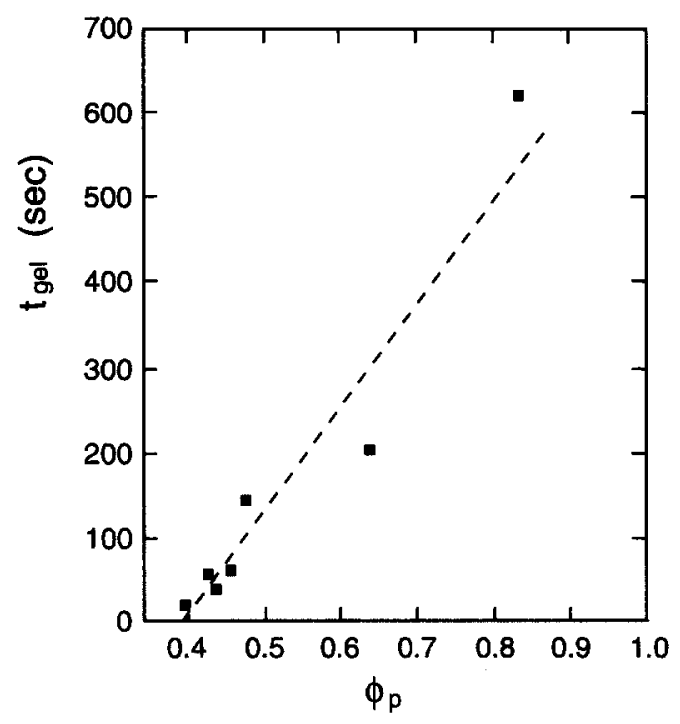

Fig. 4. Gel life time $\left(t_{\mathrm{gel}}\right)$, as determined from the time evolution of the turbidity, versus volume fraction polymer $\phi_{\mathrm{p}}\left(\phi_{\mathrm{c}} \approx 0.1\right)$. A line has been drawn through the data points with the only aim of guiding the eye.

$\alpha \approx 4$ (see Table 1). The smaller value measured for sample $F$, namely $\alpha=3.1$, was determined from a limited range in $q$. The $q^{-4}$ decay, which is known as Porod behaviour [22] is typically observed from scatterers with sharp surfaces.

The scattering data can in some cases be affected by multiple scattering. Nevertheless, we present here a qualitative interpretation of the scattering data which is mainly based on the presence of the peak at non-zero transferred momentum and on the observation of a steeper asymptotic decay (larger decay exponent $\alpha=4$ ) than expected for a fractal colloidal gel. Simulation tests [23] have shown that even at the highest beam attenuation here encountered (namely, 65\%), multiple scattering would not cause disappearance or displacement of the scattering peak. The main effect would be a broadening of the peak, resulting in an effective lower value for the decay exponent $\alpha$.

After the life time of the gel the peak at non-zero wave vector vanishes and strong forward scattering is observed, as can be seen in Fig. $3(t=90 \mathrm{~s}$ and $t=110 \mathrm{~s})$. At the same time a sharp increase in turbidity occurs (see Fig. 3(b)). The time-evolution of the turbidity provided a convenient method to determine quantitatively the lifetime of the gel, which was identified as the duration of the plateau region in the sample turbidity ( $t_{\mathrm{gel}}$, see Table 1). In Fig. 4 the gel life time is plotted versus $\phi_{\mathrm{p}}$ (at constant volume fraction colloid: $\phi_{\mathrm{c}}=0.1$ ). A longer gel life time is observed with increasing $\phi_{\mathrm{p}}$.

\subsection{Light microscopy}

In Fig. 5 we show micrographs of the mixture $\mathrm{C}\left(\phi_{\mathrm{p}}=42 \%\right.$ and $\left.\phi_{\mathrm{c}}=11 \%\right)$. All images have been recorded in the bulk of the system at the same location at different times after homogenization. Each photograph shows a pattern consisting of 

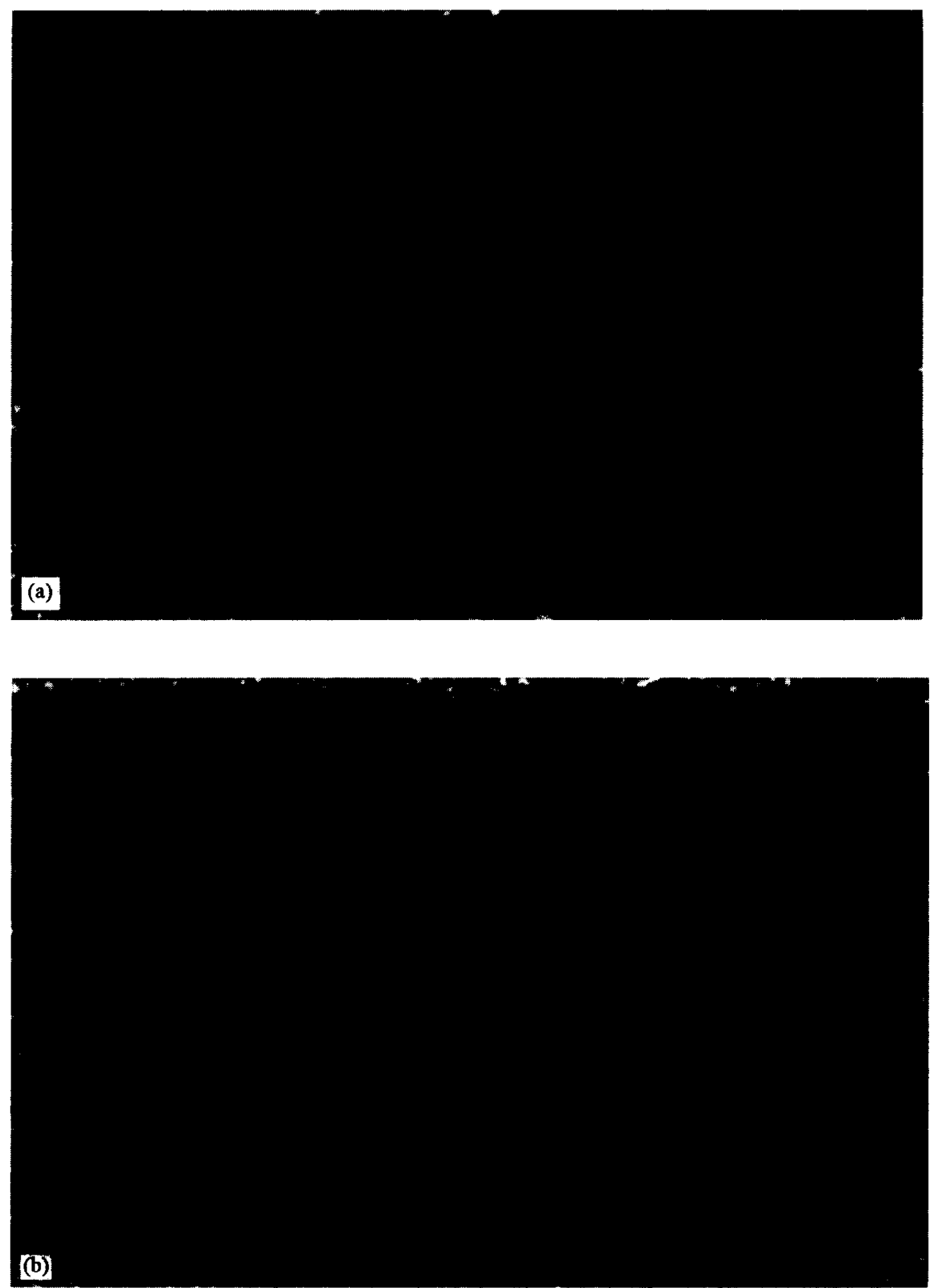

Fig. 5. Micrographs of a mixture with $\phi_{\mathrm{p}}=0.42$ and $\phi_{\mathrm{c}}=0.11$, at a sequence of times after homogenization: $5 \mathrm{~s}(\mathrm{a}), 15 \mathrm{~s}(\mathrm{~b}), 60 \mathrm{~s}(\mathrm{c})$, and $75 \mathrm{~s} \mathrm{(d).} \mathrm{The} \mathrm{bar} \mathrm{in} \mathrm{each} \mathrm{micrograph} \mathrm{equals} 100 \mu \mathrm{m}$.

bright and dark domains. Since the figures are only two dimensional slices they do not show connected domains as connected. We assume that the white spots are parts of connected domains. Fig. 5(a) has been obtained $5 \mathrm{~s}$ after homogenization, when still some motion could be observed in the system. After $15 \mathrm{~s}$ however (Fig. 5(b)), 

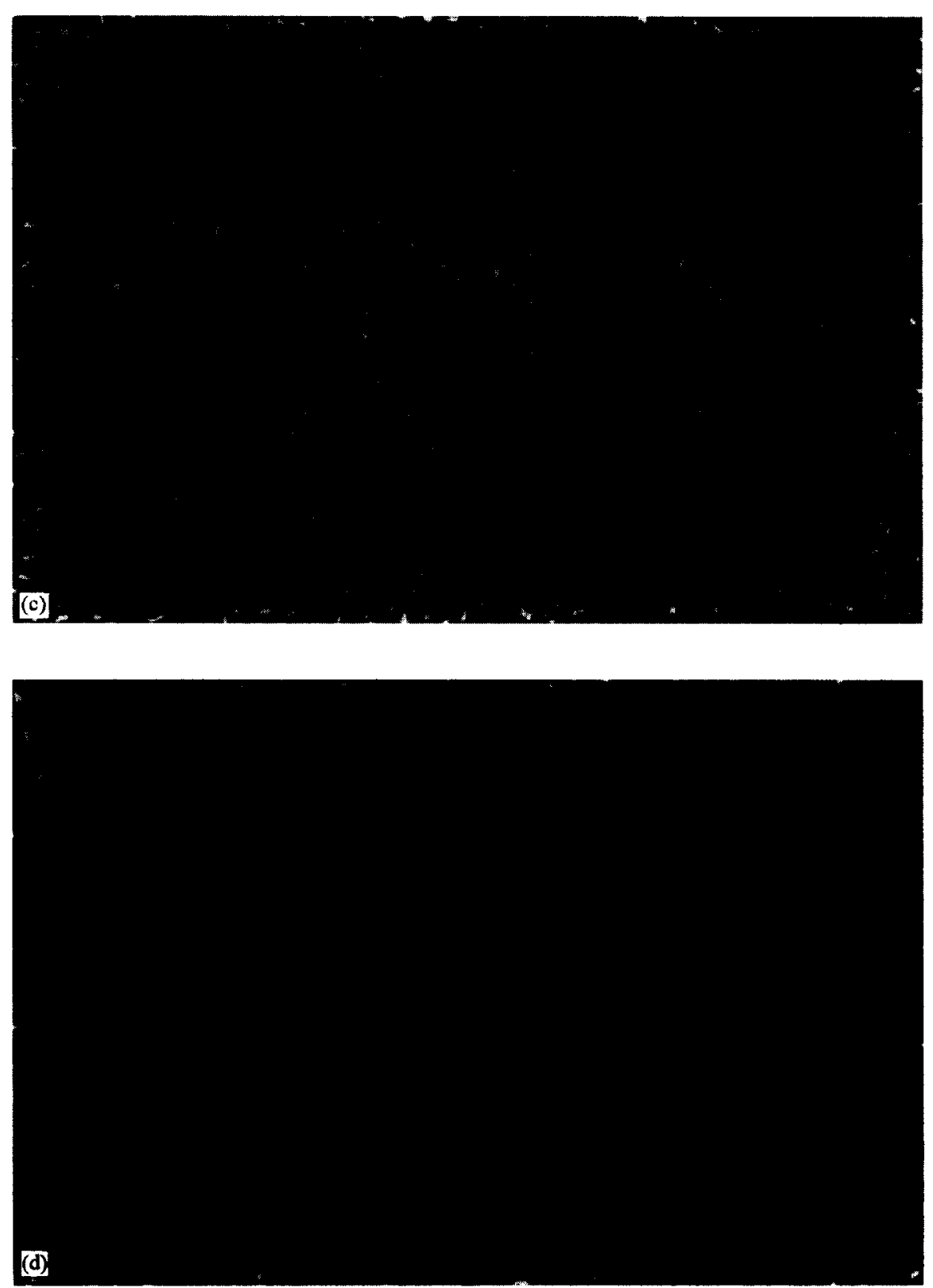

Fig. 5. Continued.

the structure appears almost frozen indicating that the system has gelled. Nevertheless, some rearrangement still takes place. Comparing Fig. 5(b) with 5(c) (60s) a slight coarsening of the domains together with an increasing contrast can be noticed. This is even more evident in Fig. 5(d) (75s) taken just before the collapse of the gel, 
when motion becomes visible again. We notice that the life time, as determined by microscopy, is in good agreement with the one obtained by turbidity measurements (see Table 1). Furthermore, the typical length scale determined via optical microscopy is in the range $10-20 \mu \mathrm{m}$, consistent with the light scattering results.

\section{Discussion}

The generally accepted model for particle gels is that individual colloidal particles aggregate into fractal clusters (with fractal dimension $d_{\mathrm{f}}$ ) which grow until they form a space spanning structure and gelation occurs. Like the constituent clusters the resulting particle gel exhibits a fractal geometry implying a $q^{-d_{f}}$ decay of the light scattering profile in the asymptotic high $q$ regime. Such a $q$ dependence has indeed been observed experimentally in permanent particle gels which are formed in dilute suspensions ( $\phi<$ $0.01)$ with irreversible bonds between the aggregating particles $[12,24]$ but also in more concentrated systems with reversible bonds $[25,26]$.

In the present work we have no experimental evidence of a fractal geometry for the particle gel, on the contrary we observe an asymptotic $q^{-4}$ decay, indicative for scatterers with smooth interfaces. Therefore the above scenario for gelation does not apply to our system. As mentioned in the Introduction gelation can also result from a fluid-fluid phase separation which is arrested at some intermediate stage either by a sol-gel transition $[13,27]$ or by a glass transition $[14,15]$ leaving the system in a state of microphase separation. A thermodynamic model for this mode of gelation was presented by Frank and Keller [28] and a kinetic model by Sciortino et al. [29]. On the basis of our experimental observations we argue that such a mechanism of gelation by phase separation appears to be applicable to the formation of transient gels in the colloid-polymer mixtures studied here. Following Refs. $[28,29]$ the envisaged situation regarding the interference between phase separation and the sol-gel transition, adapted for the case of colloid-polymer mixtures, is illustrated in Fig. 6(a). It shows schematically the (metastable) fluid-fluid phase boundary, the spinodal and the percolation line indicating the sol-gel transition. Systems with compositions in the two-phase region below the tieline $\mathrm{A}^{\prime}-\mathrm{A}^{\prime \prime}$, which just intersects with the percolation line will phase separate without being modified by the sol-gel transition. However the phase separation of systems above this tie line will be interrupted by the percolation line. As the spinodal decomposition process proceeds the percolation threshold concentration is reached inside the colloid rich regions (Fig. 6(b)). At this threshold concentration a space spanning cluster of aggregated particles can be found and the system becomes endowed with some rigidity, i.e. gelation occurs. The system is now (temporarily) prevented from proceeding towards macroscopic phase separation.

In Fig. 7 we give a schematic representation of the microscopic structure of the system during the gel life time, based on the ideas discussed above. This model allows us to explain the experimental light scattering, optical microscopy and turbidity data. First of all the bicontinuous network resulting from spinodal decomposition exhibits 

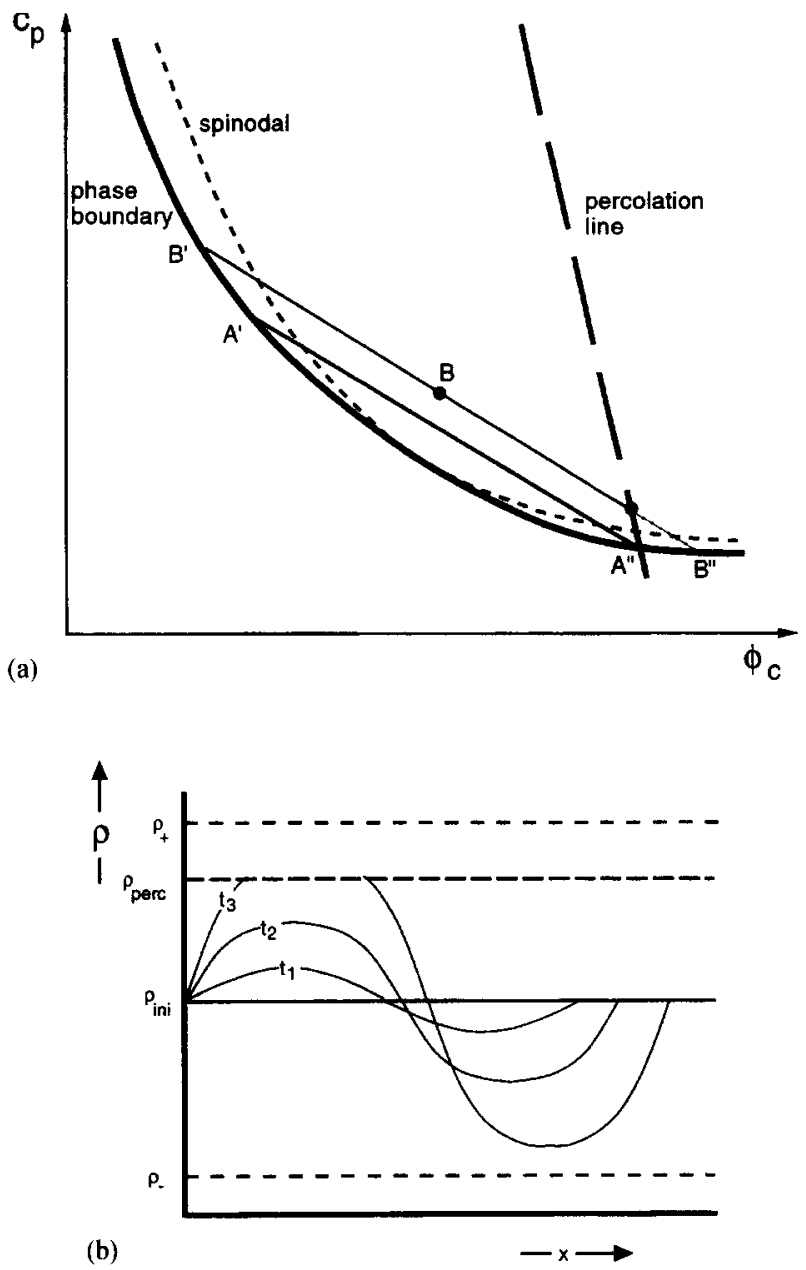

Fig. 6. (a) Schematic representation of colloid volume fraction $\left(\phi_{c}\right)$ vs. polymer concentration $\left(c_{p}\right)$ plane of the interference between phase separation and the percolation transition in phase separating colloid-polymer mixtures. The fluid-fluid phase separation line is intersected by a percolation line, the threshold concentration at which a space spanning cluster of aggregated particles can be found. Below the tie line $\mathrm{A}^{\prime}-\mathrm{A}^{\prime \prime}$ the phase separation is not affected by the percolation transition; however, above this tie line the phase separation is interrupted by the percolation transition. A system with composition B cannot reach the equilibrium composition $\mathrm{B}^{\prime \prime}$ because the tie-line cuts the percolation line; at that point in the colloid rich regions an infinite cluster of aggregated particles appears leading to gelation of the system. (b) Time evolution of colloid concentration fluctuations induced by the spinodal decomposition process. The initial homogeneous concentration is $\rho_{\text {ini }}$, the coexisting concentrations are $\rho_{+}$and $\rho_{-}$for the colloid-rich and colloid-poor phase, respectively. When the dense phase reaches a concentration $\rho_{\text {perc }}$ the colloidal particles form a percolated network in the colloid-rich phase (gel state).

quasi-ordered concentration fluctuations, characterized by a wavelength $A$ which accounts for the maximum at $q_{\mathrm{m}}$ in $I(q)$. This bicontinuous network also explains the regular distribution of bright and dark regions visible in the optical micrographs. The Porod tail $q^{-4}$ of $I(q)$, also typical or spinodal decomposition, is due to the sharp interfaces 


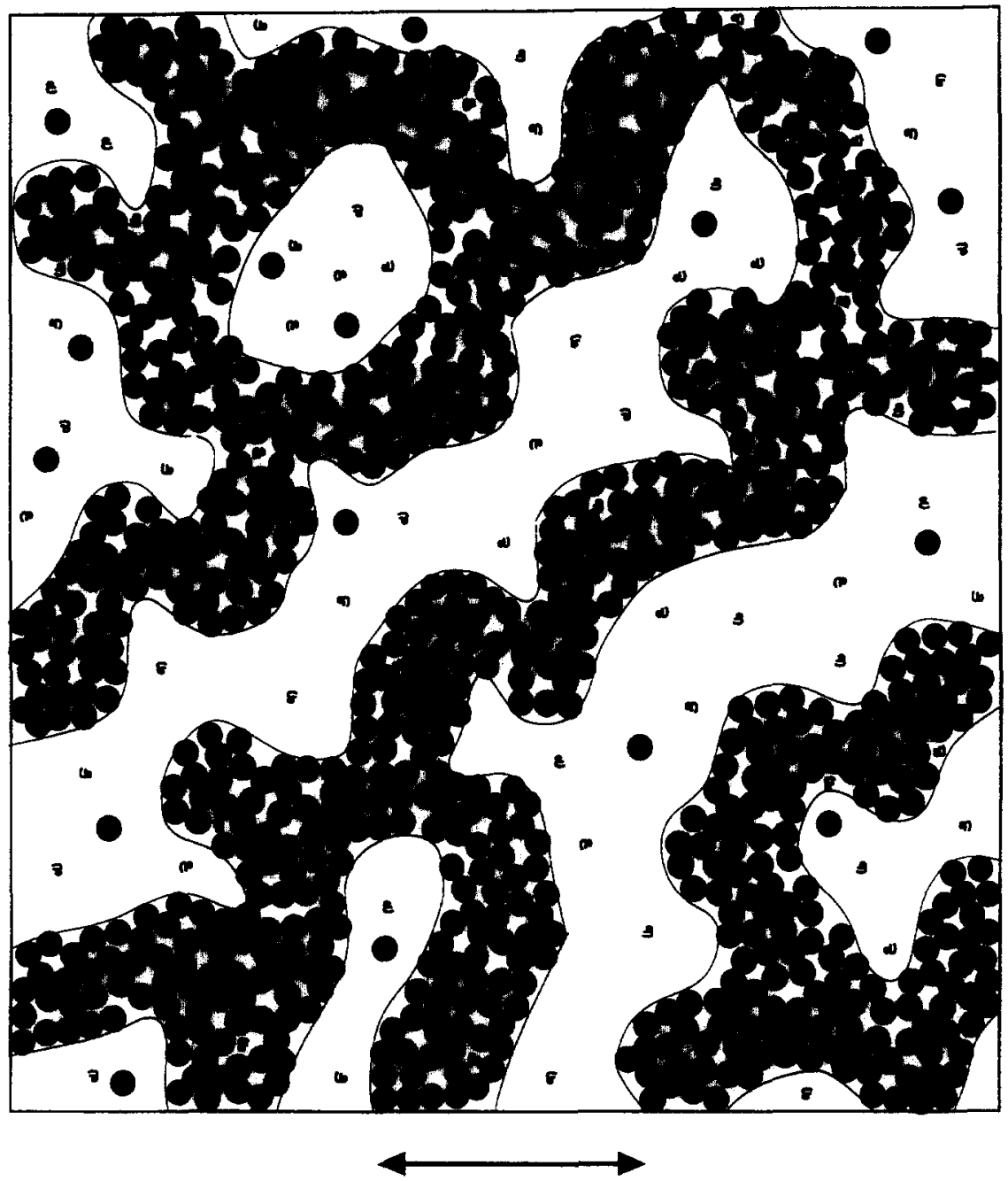

$\Lambda=2 \pi / \mathrm{q}$

Fig. 7. Schematic representation of a bicontinuous network, endowed with a characteristic length scale $A$ (wavelength of concentration fluctuations, which is inversely proportional to $q_{\mathrm{m}}$ ). Colloid-rich and colloid-poor domains are separated by sharp interfaces, explaining the Porod decay in the scattering intensity at high wave vector. The colloid-rich domains consist of a percolated colloidal structure.

between colloid-rich domains and the colloid-poor domains. Note that the Porod law is observed at $q$-vectors smaller than those expected for the scattering from the particle surfaces. Indeed, non-fractal colloidal aggregates exhibiting Porod behaviour have already been reported in reversible aggregation condition [30]. The model of spinodal decomposition driven gelation also accounts for the turbidity behaviour since the turbidity depends on the optical contrast between colloid-rich and colloid-poor domains. The turbidity increases during the gel formation because the amplitude of the concentration 
fluctuation, and thus the optical contrast, grows (Fig. 6). Accordingly, when the gel is formed, it attains a plateau value. Furthermore, when the system is macroscopically phase separated the turbidity in both phases is lower than in the initial stages of the process.

In the system under study, the potential well depth induced by the polymers is in the order of $2.5-5 k_{\mathrm{B}} T$ (see Eq. (1)), thus the bonds are reversible. Experimentally it is observed that during the gel lifetime the speckles fluctuate with time constants that are hardly $q$-dependent. This observation indicates that restructuring does not occur via diffusion of single particles, consistent with the fact that the particles are connected in the network. However, we speculate that due to the reversible nature of the bonds, the internal structure of the network continuously rearranges over all length scales within the characteristic time interval associated with unbinding. During the gel life time, the rearrangement does not strongly affect the characteristic wavelength of the concentration fluctuation, as is evidenced by the fixed position of the scattering peak. However, large scale rearrangement gradually reduces the order in the system, thus accounting for the filling of the peak.

Eventually, the restructuring leads to a loss of connection in the network, as also suggested by simulations [31-33]. Thus the polymer-induced two-phase separation phenomenon can proceed to a macroscopic extent, via the collapse of the gel. The onset of the collapse corresponds to the end of the gel life time, which increases with polymer concentration. This can be explained by a reduced rearrangement efficiency due to a deeper potential well (Eq. (1)). The collapse occurs with a sharp interface between the two phases, which is another signature of a genuine phase separation process.

\section{Conclusions}

We argued that transient gels in colloid-polymer mixtures are spinodal decomposition driven. Depletion forces induce the system to phase separate via the formation of a bicontinuous network of colloid-rich and colloid-poor domains. The presence of a space spanning colloid-rich phase with increasing colloid concentration favours the growth of a particle gel which temporarily arrests phase separation. The peak at finite angle and the Porod tail in the scattered intensity, as well as the alternating pattern of bright and dark domains in the micrographs, reveal the presence of a characteristic length scale and sharp interfaces in the bicontinuous network. We plan to investigate in more details the internal structure of the gel by means of confocal scanning laser microscopy, which is also a powerful technique for studying the complicated phenomena of restructuring and collapse of the gel.

\section{Acknowledgements}

This work was supported by the Stichting voor Fundamenteel Onderzoek der Materie (Foundation for Fundamental Research on Matter) which is part of the Nederlandse 
Organisatie voor Wetenschappelijk Onderzoek (Netherlands Organization for the Advancement of Research). The collaboration between the Milan and Utrecht groups was made possible by the financial support of the European Community for the Network Colloid Physics. We would like to thank Marina Carpineti and Jan Dhont for valuable discussions and technical support. D.A. and N.V. acknowledge fruitful stays at the Van't Hoff Laboratory and the University of Milan, respectively.

\section{References}

[1] S. Asakura, F. Oosawa, J. Chem. Phys. 22 (1954) 1255.

[2] A. Vrij, Pure Appl. Chem. 48 (1976) 471.

[3] A.P. Gast, C.K. Hall, W.B. Russel, J. Colloid Interface Sci. 96 (1983) 251.

[4] H.N.W. Lekkerkerker, W.C.K. Poon, P.N. Pusey, A. Stroobants, P.B. Warren, Europhys. Lett. 20 (6) (1992) 559.

[5] W.C.K. Poon, J.S. Selfe, M.B. Robertson, S.M. Ilett, A.D. Pirie, P.N. Pusey, J. Phys. II France 3 (1993) 1075.

[6] S.M. Ilett, A. Orrock, W.C.K. Poon, P.N. Pusey, Phys. Rev. E 51 (1995) 1344.

[7] F. Leal Calderon, J. Bibette, J. Bias, Europhys. Lett. 23 (9) (1993) 653.

[8] N.A.M. Verhaegh, J.S. van Duijneveldt, J.K.G. Dhont, H.N.W. Lekkerkerker, Physica A 230 (1996) 409.

[9] P.N. Pusey, A.D. Pirie, W.C.K. Poon, Physica A 201 (1993) 322.

[10] W.C.K. Poon, A.D. Pirie, P.N. Pusey, Faraday Discussion 101 (1995) 65.

[11] W.C.K. Poon, A.D. Pirie, M.D. Haw, P.N. Pusey, Physica A 235 (1997) 216.

[12] M. Carpineti, M. Giglio, Phys. Rev. Lett. 68 (1992) 3327.

[13] C.A. Miller, D.D. Miller, Colloids Surfaces 16 (1985) 219.

[14] J. Arnauts, H. Berghmans, Polymer Comm. 28 (1987) 66.

[15] R.M. Hikmet, S. Callister, K. Keller, Polymer 29 (1988) 1378.

[16] K. Osseo-Asare, F.J. Arriagada, Colloid Surf. 50 (1990) 321.

[17] F.J. Arriagada, K. Osseo-Asare, Colloid Surf. 69 (1992) 105.

[18] A. van Blaaderen, in press.

[19] A.K. van Helden, J.W. Jansen, A. Vrij, J. Colloid Interface Sci. 77 (2) (1980) 418.

[20] M. Carpineti, M. Giglio, F. Ferri, E. Paganini, U. Perini, Phys. Rev. A 42 (1990) 7347.

[21] H. Verduin, J.K.G. Dhont, J. Colloid Interface Sci. 172 (1995) 425.

[22] G. Porod, in: O. Glatter, O. Kratky (Eds.), Small Angle X-ray Scattering, Academic Press, London, 1982.

[23] L. Cipelletti, PhD. Thesis, University of Milan, 1996.

[24] G. Dietler, C. Aubert, D.S. Cannell, P. Wiltzius, Phys. Rev. Lett. 57 (1986) 3117.

[25] P.W. Rouw, C.G. de Kruif, Phys. Rev. A 39 (1989) 5399.

[26] R. Piazza, G. Dipietro, Europhys. Lett. 28 (1994) 445.

[27] D. Asnaghi, M. Giglio, A. Bossi, P.G. Righetti, J. Chem. Phys. 102 (1995) 9736.

[28] F.C. Frank, A. Keller, Polymer Commun. 29 (1988) 186.

[29] F. Sciortino, R. Bansil, H.E. Stanley, P. Alstrom, Phys. Rev. E 47 (1993) 4615.

[30] M.L. Broide, Y. Garrabos, D. Beysens, Phys. Rev. E 47 (1993) 3768.

[31] M.D. Haw, M. Sievwright, W.C.K. Poon, P.N. Pusey, Adv. Colloid Interface Sci. 62 (1995) 1.

[32] M.T.A. Bos, J.H.J. van Opheusden, Phys. Rev. E 53 (1996) 5044.

[33] B.H. Bijsterbosch, M.T.A. Bos, E. Dickinson, J.H.J. van Opheusden, P. Walstra, Faraday Discussion 101 (1995) 51 